\title{
Agents That Negotiate Proficiently with People
}

\author{
Sarit Kraus \\ Dept. of Computer Science Bar-Ilan University, Ramat Gan 52900 Israel and \\ Institute for Advanced Computer Studies University of Maryland, MD 20742 USA \\ sariteumiacs.umd.edu
}

\begin{abstract}
Negotiation is a process by which interested parties confer with the aim of reaching agreements. The dissemination of technologies such as the Internet has created opportunities for computer agents to negotiate with people, despite being distributed geographically and in time. The inclusion of people presents novel problems for the design of autonomous agent negotiation strategies. People do not adhere to the optimal, monolithic strategies that can be derived analytically, as is the case in settings comprising computer agents alone. Their negotiation behavior is affected by a multitude of social and psychological factors, such as social attributes that influence negotiation deals (e.g., social welfare, inequity aversion) and traits of individual negotiators (e.g., altruism, trustworthiness, helpfulness). Furthermore, culture plays an important role in their decision making and people of varying cultures differ in the way they make offers and fulfill their commitments in negotiation.

In this talk I will present the following two agents that negotiate well with people by modeling several social factors: The PURB agent that can adapt successfully to people from different cultures in complete information settings, and the SIGAL agent that learns to negotiate successfully with people in games where people can choose to reveal private information. These agents were evaluated in extensive experiments including people from three countries. I will also demonstrate how agents' opponent modeling of people can be improved by using models from the social sciences.
\end{abstract}

Acknowledgements. This research is based upon work supported in part by the U.S. Army Research Laboratory and the U.S. Army Research Office under grant number W911NF-08-1-0144 and under NSF grant 0705587.

\section{References}

1. Gal, Y., Grosz, B., Kraus, S., Pfeffer, A., Shieber, S.: A framework for Investigating Decision-Making in Open Mixed Networks. Artificial Intelligence Journal 174(18), 1460-1480 (2010)

2. Gal, Y., Kraus, S., Gelfand, M., Khashan, H., Salmon, E.: Negotiating with People across Cultures using an Adaptive Agent. ACM Transaction on Intelligent Systems and Technology (2011)

3. Peled, N., Kraus, S., Gal, K.: A Study of Computational and Human Strategies in Revelation Games. In: Proc. of AAMAS 2011 (2011) 